\title{
Hospital Preparedness for Critical Care during COVID-19 Pandemic: Exploratory Cross-sectional Study
}

\author{
John Rene Labib ${ }^{1}$, Sally Kamal ${ }^{1}$, Marwa Rashad Salem², Eman D. El Desouky ${ }^{3 *}$, Ahmed Taher Mahmoud $^{4}$ \\ ${ }^{1}$ Department of Pediatrics, Faculty of Medicine, Cairo University, Cairo, Egypt; ${ }^{2}$ Department of Public Health and Community \\ Medicine, Faculty of Medicine, Cairo University, Cairo, Egypt; ${ }^{3}$ Department of Epidemiology and Biostatistics, National Cancer \\ Institute, Cairo University, Cairo, Egypt; ${ }^{4}$ Department of Critical Care Medicine, Faculty of Medicine, Cairo University, Cairo, \\ Egypt
}

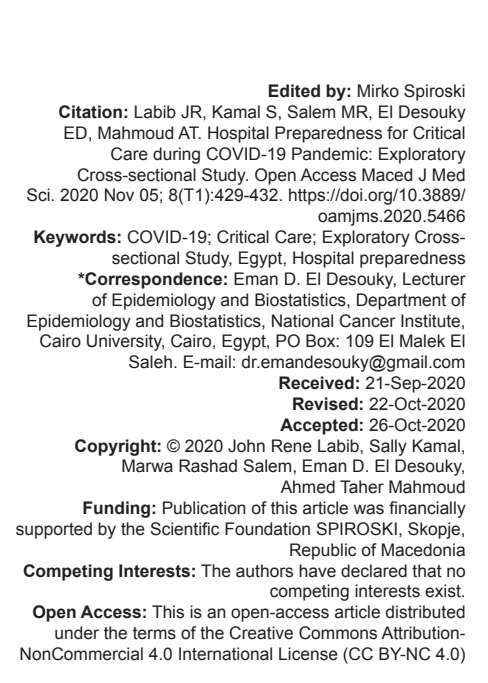

\section{Introduction}

Coronavirus disease 2019 (COVID-19) caused by severe acute respiratory syndrome coronavirus (SARS-CoV-2) has promptly turned to a universal pandemic [1]. A high incidence of acute respiratory distress syndrome (ARDS) [2] and critical illness (23-32\%) among hospitalized patients was reported worldwide [3]. In Egypt, similar rates of critical illness (84\%) were also reported [4]. The reported Intensive care units (ICU) mortality rates are alarmingly high [5]; deaths from COVID-19 has already far exceeded the combined deaths from MERS and SARS [6]. During a pandemic, challenging situation is experienced mandating of increasing the capacity of ICU and the fact that critically ill patients might need to get care outside the traditional ICU [5]. The progressive spread of disease during an outbreak can overwhelm hospitals' ability to respond, as there are too many patients needing medical care at the same time. Intensive care units were concurrently challenged on numerous aspects. These include resource limitations, infection control, protection of healthcare workers ( $\mathrm{HCWs}$ ), and adaptation of services to a rapidly evolving pandemic situation. During the early phase of the outbreak in Wuhan, China, shortages in equipment meant that $75 \%$ of the deceased did not receive mechanical ventilation [7]. ICU resources in Egypt are also reported to be overwhelmed [4]. Intensive care units should prioritize and implement actions specified in their emergency preparedness plans for COVID-19 to identify suspected cases, limit transmission within the facility, and provide specialized medical care. This includes activating protocols and procedures in safe physical spaces, emphasizing isolation measures, education, and training of personnel in the use of personal protective equipment (PPE), patient management, sample collection and handling, and handling and disposal of hazardous biological waste. Consequently, the researchers conduct the current exploratory crosssectional study to assess ICUs preparedness in Cairo 
University Hospitals to deal efficiently and effectively with COVID-19 upcoming waves.

\section{Methods}

\section{Study type and study setting}

An exploratory cross-sectional study was conducted at Cairo University Intensive Care Units(6 pediatrics ICUs and 2 adult ICUs) in the period from end of February to the $1^{\text {st }}$ week of March, 2020; almost 2 weeks after the appearance of the first case of COVID-19 in Egypt by hand-delivered questionnaire methodology with one of the staff members who were available and have time to take part in the study.

\section{Data collection tool}

WHO checklist for hospital readiness was used; this checklist based on current knowledge and available evidence on the COVID-19 pandemic for WHO's Regional Office for the Eastern Mediterranean Region. This checklist has been developed to help hospital managers prepare for COVID-19 patient management by optimizing each hospital's capacities [8]. The list composed of 10 key components: (1) Leadership and coordination; (2) operational support, logistics and supply management; (3) information; (4) communication; (5) human resources; (6) continuity of essential services and surge capacity; (7) rapid identification; (8) diagnosis; (9) isolation and case management; and (10) infection prevention and control (Figure 1).

\section{Statistical analysis}

The researchers did the statistical analysis using the EXCEL program version 10. Each component consisted of several response readiness activities with answers: Yes, in progress and no; this transformed to $1,0.5$, and 0 points, respectively. All points (scores) in each component were summed up to total score per component, then overall percent readiness was calculated as; Component readiness percentage $=$ (summation of achieved scores/total maximum possible score $)^{*} 100$. The higher the percentage was the better readiness. They calculated average ICU readiness and overall average for each component on the summed score and percentage also calculated for pediatrics and adults.

\section{Ethical considerations}

Approval of the study protocol was obtained from the Ethical Committee of the Faculty of Medicine,

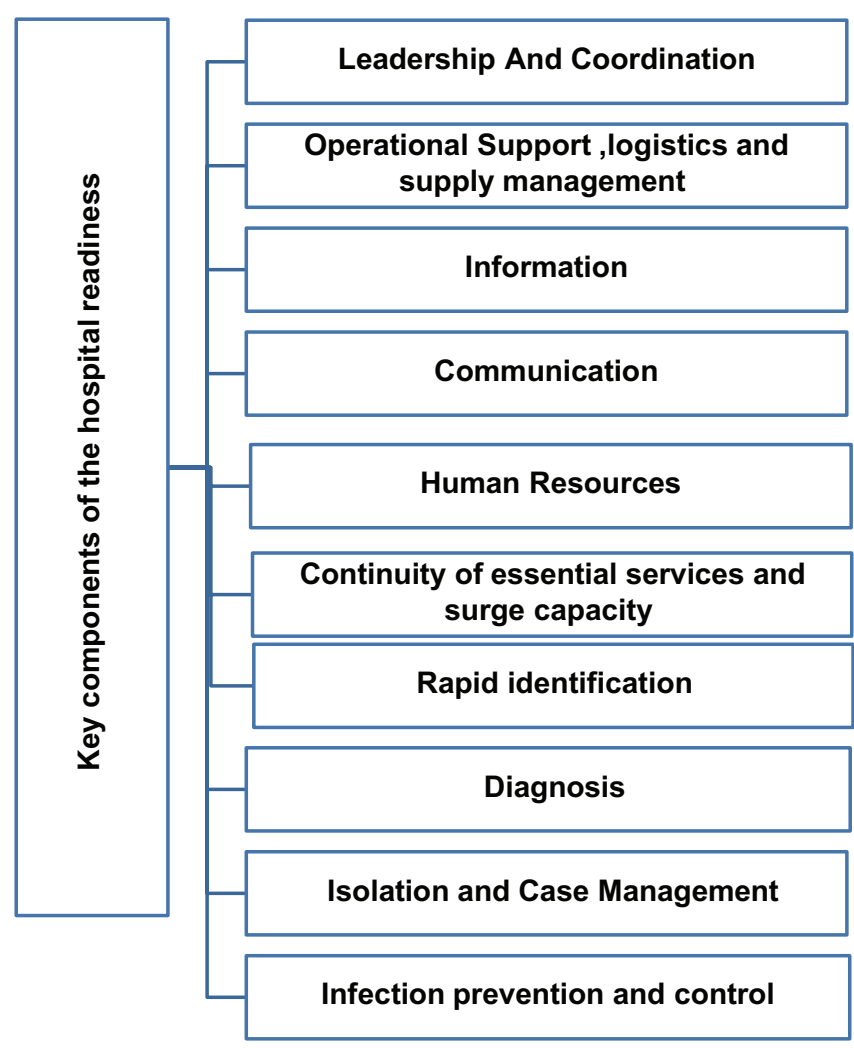

Figure 1: Key components of the hospital readiness checklist for COVID-19

Cairo University. Confidentiality of data, safe data storage, and privacy rights had been respected. Only those who agreed were included, and those who refused were excluded from the study. All procedures for data collection were treated with confidentiality according to the Helsinki declarations of biomedical ethics [9]. Results of the survey for every ICU were sent to the participant staff member to evaluate the current status of ICU readiness.

\section{Results}

The facilities screened were all related to Cairo University, which is public hospitals linked to the Ministry of Higher Education. All hospitals had diagnostic facilities such as the laboratory, X-ray, CT, and MRI. The overall preparedness in both pediatric and adult ICUs was 54\% (Table 1, Figure 2).

Table 1: Overall pediatric and adult Cairo university ICU readiness for COVID-19

\begin{tabular}{llll}
\hline Component & Overall (\%) & Pediatric & Adult \\
\hline 1. Leadership and coordination & 71 & 68 & 82 \\
2. Operational support, logistics, and supply management & 75 & 76 & 75 \\
3. Information & 28 & 33 & 10 \\
4. Communication & 43 & 38 & 60 \\
5. Human resources & 28 & 28 & 29 \\
6. Continuity of essential services and surge capacity & 60 & 55 & 75 \\
7. Rapid identification & 61 & 57 & 72 \\
8. Diagnosis & 61 & 54 & 83 \\
9. Isolation and case management & 47 & 35 & 86 \\
10. Infection prevention and control & 68 & 65 & 75 \\
Overall & 54 & 51 & 65 \\
\hline
\end{tabular}




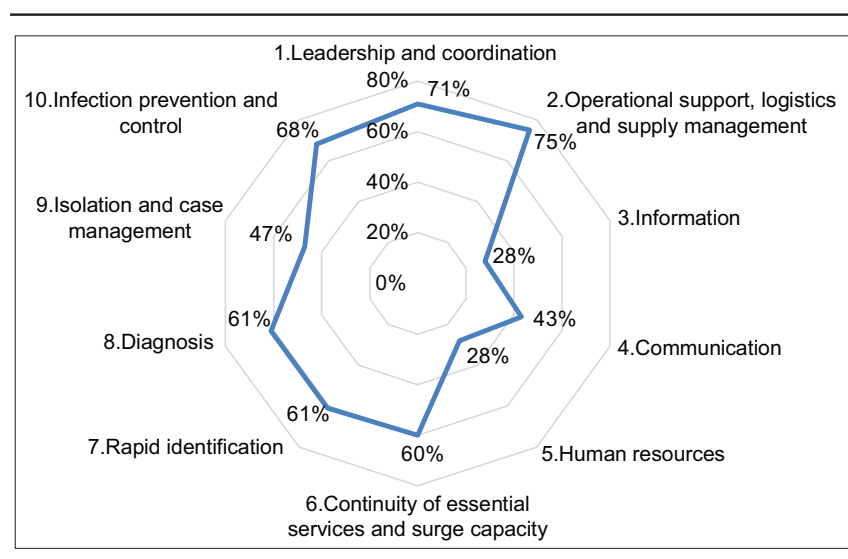

Figure 2: Overview of hospital readiness: Key components

Overall, adult ICUs were more prepared than pediatric ICUs, especially in communication; continuity of essential services and surge capacity; rapid identification; diagnosis; isolation; and case management. Both of them were comparable regarding operational support, logistics, and supply management; human resources; and infection prevention and control, while information component was lower in both types but reached critical values $10 \%$ in adult ones as displayed in Table 1.

\section{DISCUSSION}

Researchers conducted the current exploratory study to explore the readiness of Cairo University ICUs to deal with the current COVID-19 pandemic and the upcoming waves, the study revealed that the overall preparedness in both pediatric and adult ICUs was $54 \%$. This was an alarming figure because of the high ICU bed occupancy rates as reported by the Minister of Health and Population in Egypt (84\%) by COVID-19 patients according to the latest figures released from the Ministry of Health and Population in Egypt [4].

The study showed that adult ICUs were more prepared than pediatric ones; this might be due to the current belief of COVID-19 is mainly affect adults more than pediatrics.

The overall prevention and control component achieved $65 \%$ only. For the safety of hospital personnel and the prevention of nosocomial infections of COVID-19, training and strict adherence to infection control measures recommended by national and international bodies are of great importance. Training on-site and periodic refresher re-training are required to guarantee staff readiness and proficiency with a particular emphasis on personal protective equipment (PPE) [10], [11]. In Italy, up to $20 \% \mathrm{HCW}$ have also been confirmed to be infected doing their work at COVID hospitals[12], unsurprising that concern regarding human resources where it is fulfilled by $28 \%$ only. Particularly outside of the pandemic, there is an insufficient supply of qualified nursing staff and intensive care physicians. A short-term increase in skilled personnel, which would be appropriate to intensely increase intensive care capability, even outside of the pandemic, the availability of trained nursing staff and doctors in ICUs is limited worldwide [4], [5]. Therefore, a short-term increase in qualified staff, which would be essential to significantly increase intensive care capacities, is unrealistic. Unconventional ideas for recruitment must be considered early, like to draw manpower from different departments and divisions in the hospital with the application of the needed training.

The study detected a defect in communications; this reflects the need for the presence of open communication channels and rapid distribution of information to keep staff informed of new developments.

We should view the current study finding in accordance with the following limitation that this study was limited to one hospital, so its findings may not be generalizable to other hospitals; however, the researchers conducted the current study to explore the situation in this new area of inquiry; COVID-19 pandemic.

\section{Conclusion}

The current study demonstrated the intermediate readiness of ICUs at initial outbreak; further assessment during different phases of pandemic is required. Continues education of HCWs on infection control measures is required until the pandemic fenced. Active communication is vital in pandemic response. Information should be circulated to all HCWs in a timely fashion, and two-way communication should be established online disaster management plans, protocols for identification and isolation had to be placed in place to sustain medical treatment or at least maintain emergency care. Increasing in ICU bed capacity is mandatory.

\section{Acknowledgments}

The authors are thankful to the Chairmen of the intensive care units for conducting this study. The authors also thank the study participants for their active participation in the present study. 


\section{References}

1. Meo SA, Alhowikan AM, Al-Khlaiwi T, Meo IM, Halepoto DM, Iqbal M, et al. Novel coronavirus 2019-nCoV: Prevalence, biological and clinical characteristics comparison with SARS-CoV and MERS-CoV. Eur Rev Med Pharmacol Sci. 2020;24(4):2012-9. https://doi.org/10.26355/eurrev_202002_20379

PMid:32141570

2. Singhal T. A review of coronavirus disease-2019 (COVID-19). Indian J Pediatr. 2020;87(4):281-6. https://doi.org/10.1007/ s12098-020-03263-6

PMid:32166607

3. Hick JL, Biddinger PD. Novel coronavirus and old lessonspreparing the health system for the pandemic. N Engl J Med. 2020;382(20):e55. https://doi.org/10.1056/NEJMp2005118 PMid:32212515

4. Available from: https://www.healthresearchweb.org/en/egypt/ institution_54. [Last accessed on 2020 Sep 14].

5. Yang $X, Y u$ Y, Xu J, Shu H, Xia J, Liu H, et al. Clinical course and outcomes of critically ill patients with SARS-CoV-2 pneumonia in Wuhan, China: A single-centered, retrospective, observational study. Lancet Respir Med. 2020;8(5):475-81. https://doi. org/10.1016/S2213-2600(20)30079-5

PMid:32105632

6. Park M, Thwaites RS, Openshaw PJ. COVID-19: Lessons from SARS and MERS. Eur J Immunol. 2020;50:308-11.

7. Xie J, Tong Z, Guan X, Du B, Qiu H, Slutsky AS. Critical care crisis and some recommendations during the COVID-19 epidemic in China. Intensive Care Med. 2020;46(5):837-40. https://doi.org/10.1007/s00134-020-05979-7

PMid:32123994

8. World Health Organization. Rapid Hospital Readiness Checklist for COVID-19. Geneva: World Health Organization; 2020. Available from: https://www.apps.who.int/iris/ handle/10665/332778.

9. World Medical Association. World Medical Association declaration of Helsinki: Ethical principles for medical research involving human subjects. JAMA. 2013;310(20):2191-4.

PMid:24141714

10. Christensen L, Rasmussen CS, Benfield T, Franc JM. A randomized trial of instructor-led training versus video lesson in training health care providers in proper donning and doffing of personal protective equipment. Disaster Med Public Health Prep. 2020;30:1-15. PMid:32223776

11. Verbeek JH, Rajamaki B, Iljaz S, Sauni R, Toomey E, Blackwood B, Tikka C, Ruotslanien JH, Balci K. Personal protective equipment for preventing highly infectious diseases due to exposure to contaminated body fluids in healthcare staff. Cochrane Database Syst Rev. 2020;4:CD011621. https://doi. org/10.1002/14651858.CD011621.pub4 PMid:27093058

12. Lapolla P, Mingoli A, Lee R. Deaths from COVID-19 in healthcare workers in Italy-What can we learn? Infect Control Hosp Epidemiol. 2020;???:1-2. https://doi.org/10.1017/ice.2020.241 PMid:32408922 